\title{
Acute Myeloid Leukemia-Cuplike: Characteristic Peroxidase Staining Pattern
}

\author{
Akira Okano, ${ }^{\mathrm{a}}$ Tohru Inaba ${ }^{\mathrm{b}}$ \\ ${ }^{a}$ Department of Internal Medicine, National Hospital Organization Shiga Hospital, Shiga, Japan; ${ }^{b}$ Department of \\ Molecular Genetics and Laboratory Medicine, Kyoto Prefectural University of Medicine, Kyoto, Japan
}

Received November 17, 2006; received in revised form December 14, 2006; accepted January 23, 2007

Int J Hematol. 2007;85:185. doi: 10.1532/IJH97.06218

(C) 2007 The Japanese Society of Hematology

A 71-year-old woman was admitted to the hospital because of general fatigue. She showed petechiae on her legs but showed neither hepatomegaly nor lymph node swellings. Laboratory findings were as follows: hemoglobin, $9.6 \mathrm{~g} / \mathrm{dL}$; platelets, $33 \times 10^{9} / \mathrm{L}$; white blood cells, $330 \times 10^{9} / \mathrm{L}$ with $97 \%$ leukemic blasts. The bone marrow was extremely hypercellular and almost exclusively consisted of leukemic blasts. These blasts were medium to large in size with a high nucleus-cytoplasm ratio. Fine azure granules were rarely found, but Auer bodies were not definitely identified. Interestingly, approximately $20 \%$ of these blasts seemed to have a round pale substance in the center of the nuclei, sometimes resembling prominent large nucleoli (Figure 1A). These leukemic cells were positive for myeloperoxidase staining and typically showed a crater-like appearance (Figure 1B). Immunophenotypically, the cells were CD2-, $\mathrm{CD}^{-}, \mathrm{CD}^{-}, \mathrm{CD}^{-}, \mathrm{CD}^{-}{ }^{-}, \mathrm{CD}^{+}{ }^{+}, \mathrm{CD} 19^{-}, \mathrm{CD}^{-} 0^{-}, \mathrm{CD}^{+}{ }^{+}$, $\mathrm{CD}^{-} 4^{-}, \mathrm{CD}_{56}{ }^{+}, \mathrm{CD} 117^{-}$, and HLA-DR ${ }^{-}$. From these findings, we diagnosed acute myeloid leukemia-cuplike in this patient, in accordance with the report of Kussick et al [1]. A chromosomal analysis revealed a 46,XX karyotype, but we did not examine whether internal tandem duplication of the Flt3 gene had occurred. The patient died of cerebral hemorrhage on the day following admission.

Correspondence and reprint requests: Tohru Inaba, $\mathrm{MD}, \mathrm{PhD}$, Department of Molecular Genetics and Laboratory Medicine, Kyoto Prefectural University of Medicine, Kawaramachi-Hirokoji, Kamigyo-ku, Kyoto 602-8566, Japan; 81-75-251-5652; fax: 81-75251-5678 (e-mail: inaba178@koto.kpu-m.ac.jp).
A

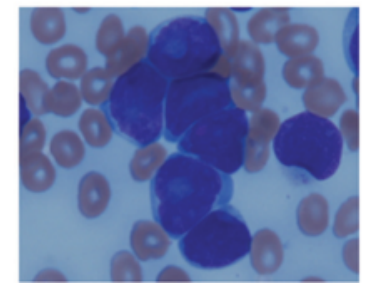

B

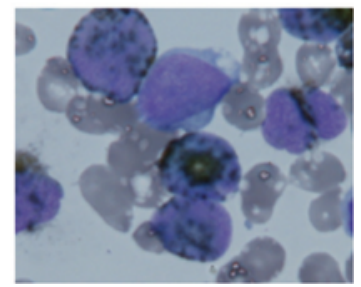

Figure 1. Leukemic cells in bone marrow at diagnosis. Wright-Giemsa staining (A) and myeloperoxidase staining (B).

Kussick et al reported a unique subset of acute myeloid leukemia morphologically characterized by a cuplike nuclear indentation in $\geq 10 \%$ of the leukemic blasts, although distinguishing such nuclear indentations from the prominent nucleoli by means of Romanowsky staining seemed difficult in certain cases. In such an instance, conventional myeloperoxidase staining revealing typical dense deposits at the centers of these cells might become a convenient diagnostic tool, as in our case.

\section{Reference}

1. Kussick SJ, Stirewalt DL, Yi HS, et al. A distinctive nuclear morphology in acute myeloid leukemia is strongly associated with loss of HLA-DR expression and FLT3 internal tandem duplication. Leukemia. 2004;18:1591-1598. 